\title{
Characteristics and roles of SARS-CoV-2 specific antibodies in patients with different severities of COVID-19
}

\author{
Zhifeng Huang ${ }^{1}$, Hao Chen ${ }^{1}$, Mingshan Xue ${ }^{1}$, Huimin Huang ${ }^{2}$, Peiyan Zheng ${ }^{2}$, Wenting \\ Luo $^{1}$, Xueqing Liang ${ }^{1}$, Baoqing Sun ${ }^{1}$, and Nanshan Zhong ${ }^{1}$ \\ ${ }^{1}$ Guangzhou Medical University \\ ${ }^{2}$ First Affiliated Hospital of Guangzhou Medical College
}

May 18, 2020

\begin{abstract}
Abstract: Background: The diagnosis of COVID-19 relies mainly on viral nucleic acid detection, but false negatives can lead to missed diagnosis and misdiagnosis. SARS-CoV-2-specific antibody detection is convenient, safe, and highly sensitive. IgM and IgG are commonly used to serologically diagnose COVID-19; however, the role of IgA is not well known. We aimed to quantify the levels of SARS-CoV-2-specific IgM, IgA, and IgG antibodies, identify changes in them based on COVID-19 severity, and establish the significance of combined antibody detection. Methods: COVID-19 patients, divided into a severe \& critical group and a moderate group, and non-COVID-19 patients with respiratory disease were included in this study. A chemiluminescence method was used to detect the levels of SARS-CoV-2-specific IgM, IgA, and IgG in the blood samples from the three groups. Epidemiological characteristics, symptoms, blood test results, and other data were recorded for all patients. Results: Compared to the traditional IgM-IgG combined antibodies, IgA-IgG combined antibodies are better for diagnosing COVID-19. During the disease process, IgA appeared first and disappeared last. All three antibodies had significantly higher levels in COVID-19 patients than in non-COVID-19 patients. IgA and IgG were also higher for severe \& critical disease than for moderate disease. All antibodies were at or near low levels at the time of tracheal extubation in critical patients. Conclusions: Detection of SARS-CoV-2-specific combined IgA-IgG antibodies is advantageous in diagnosing COVID-19. IgA detection is suitable during early and late stages of the disease. IgA and IgG levels correspond to disease severity.
\end{abstract}

\section{Hosted file}

Manuscript.doc available at https://authorea.com/users/323331/articles/452069characteristics-and-roles-of-sars-cov-2-specific-antibodies-in-patients-with-differentseverities-of-covid-19
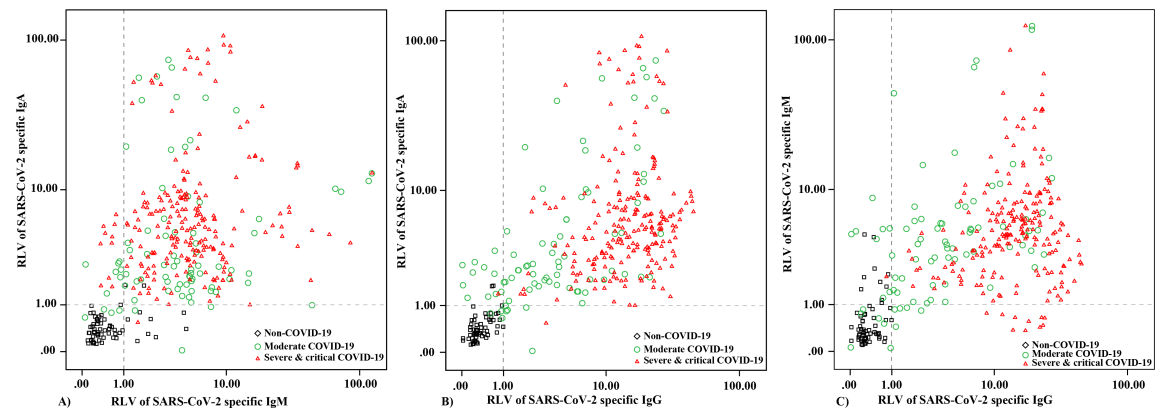

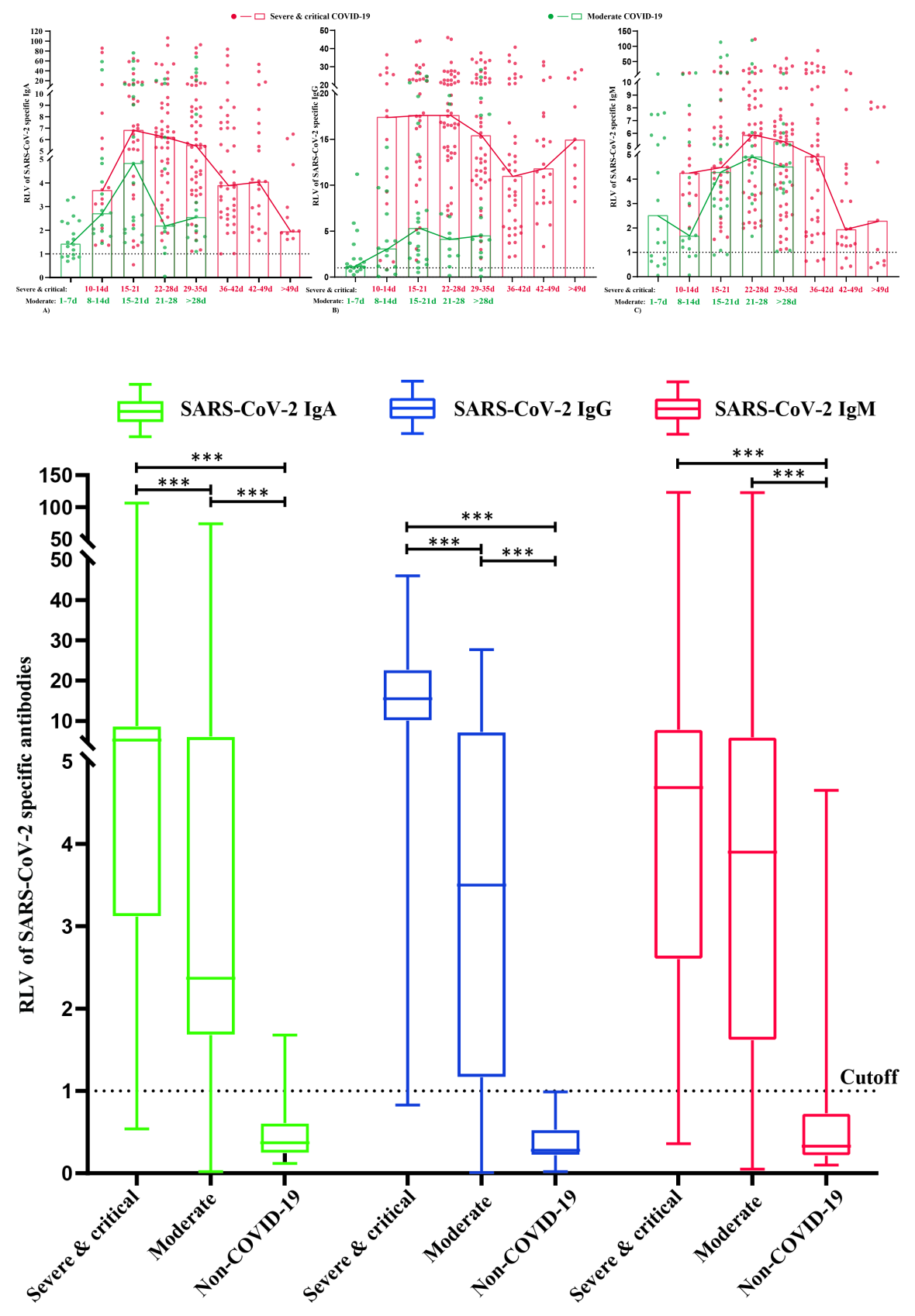


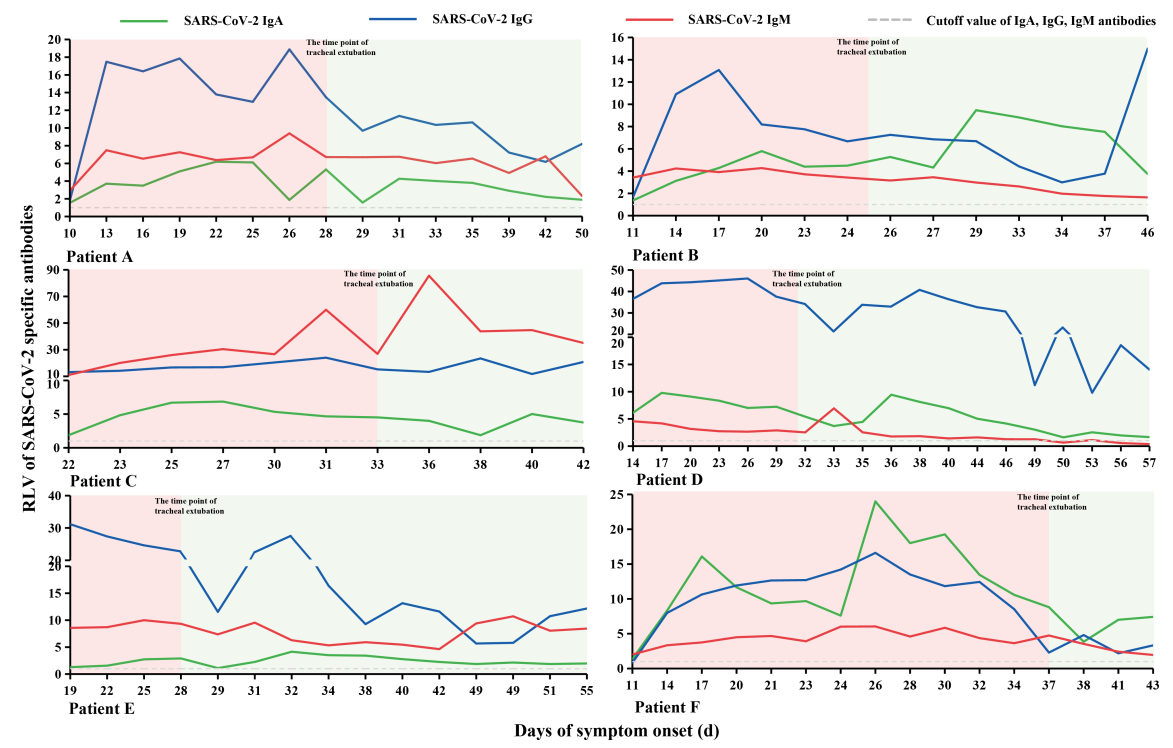

